\title{
Doxycycline and Co-trimethoxazole: A new combination for treatment of MDR Acinetobacter baumannii. Does it work?
}

\author{
S. Farid ${ }^{1}$, A. Abouelela ${ }^{2 *}$ and M. Eliwa ${ }^{2}$ \\ ${ }^{1}$ Alexandria University, Microbiology \& Immunology, Alexandria, Egypt \\ ${ }^{2}$ AlexandriaUniversity, Critical Care Medicine, Alexandria, Egypt
}

*Corresponding author

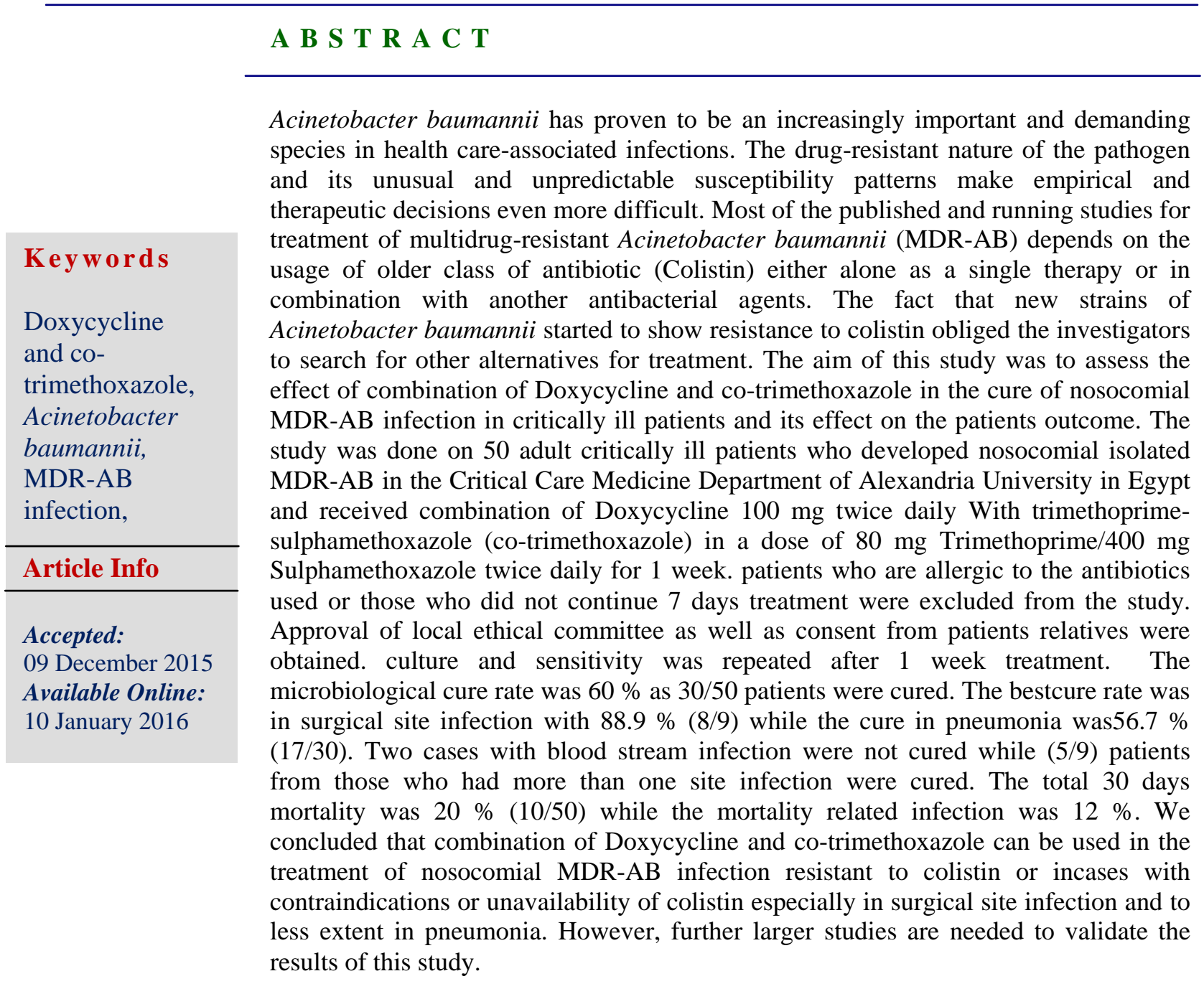




\section{Introduction}

The genus Acinetobacter was first isolated and described in 1911 by a Dutch microbiologist, several changes was introduced into the taxonomic classification of Acinetobacter species. Acinetobacter, family Moraxellaceae, consists of gramnegative bacilli that initially encompassed a heterogeneous collection of non-pigmented, oxidase-positive, and oxidase-negative gram-negative rods. It currently comprises gram-negative coccobacilli that are nonmotile, non-fermentative, non-spore forming, strictly aerobic, catalase-positive, and oxidase-negative. ${ }^{(1)}$ Acinetobacter classified as an opportunistic pathogen of relatively Low pathogencity.The organism can survive under dry conditions. Colonization in the lung is facilitated by the ability of Acinetobacter species to adhere to human bronchial epithelial cells using fimbria. ${ }^{(1,2)}$

Acinetobacter baumannii has also been isolated from a number of sites in the hospital: as environmental surfaces, equipment including mechanical ventilators and dialysis machines, air ventilation systems, water sources,hands of healthcare workers, respiratory, and gastrointestinal tracts secretions,wounds of hospitalized patients. ${ }^{(2,3)}$ Members of the genus Acinetobacter first began to be recognized as significant nosocomial pathogens during 1970. Nosocomial transmission is responsible for the vast majority of Acinetobacter infections. ${ }^{(3)}$

Although wide variations exist in different countries, there has been a general trend of increasing incidence of Acinetobacter infections. Acinetobacter was the fourth most frequently isolated respiratory pathogen, accounting for 9.6 percent of isolated bacterial species. Higher rates of Acinetobacter as a cause of nosocomial infection (27 to 35 percent of isolates) have been reported. ${ }^{(4-5)}$ In addition to sporadic infections, Acinetobacter has the potential of causing outbreaks in the hospital setting. Two important factors that contribute to the propensity for outbreaks are antimicrobial resistance and tolerance of desiccation. ${ }^{(3)}$ Most outbreaks have occurred in adult ICUs, although other hospital wards that house patients with weakened immunity are also at risk, including intensive care nurseries and burn units. Transient hand colonization among healthcare workers can contribute to outbreak dissemination. ${ }^{(5,6)}$

Treatment of bloodstream infection due to Acinetobacter consists of antibiotic therapy and intravascular catheter removal. Empiric antibiotic therapy should be selected based on local susceptibility patterns; in general, it should consist of a broad-spectrum cephalosporin, a combination betalactam/beta-lactamase inhibitor (eg, a combination including sulbactam), or a Carbapenems - either alone or in combination with an antipseudomonal fluoroquinolone or an aminoglycoside. Carbapenems monotherapy is reasonable in institutions where Carbapenems resistance rates are low; there is no evidence for improved outcome with combination therapy. ${ }^{(28-30)}$ In the setting of resistance to the above agents, therapeutic options are limited; options include colistin, polymyxin $\mathrm{B}$, and Tigecycline (only for secondary bacteremia). In one report, treatment with colistin did not reduce mortality in patients with bacteremia due to multidrug-resistant Acinetobacter. These findings highlight the poor outcome of such patients, regardless of whether treatment for bacteremia is initiated. $(7,8)$ 
The challenges of treating multidrugresistant bacteria continue to be at the forefront of the clinician's practice in caring for hospitalized patients. Acinetobacter baumannii has proven to be an increasingly important and demanding species in health care associated infections. The drug resistant nature of the pathogen and its unusual and unpredictable susceptibility patterns make empirical and therapeutic decisions even more difficult. The association of A. baumannii with pneumonia, bacteremia, wound infections, urinary tract infections, and meningitis has been well described ${ }^{(9)}$. Risk factors associated with colonization or infection (which can be difficult to distinguish) include prolonged hospitalization, intensive care unit admission, recent surgical procedures, antimicrobial agent exposure, central venous catheter use, prior hospitalization, nursing home residence, and local colonization pressure on susceptible patients ${ }^{(10-11)}$.

The particular resistance pattern displayed by Acinetobacter baumannii strains is a result of the combination between its intrinsic resistance and acquisition of resistance genetic determinants over time, most probably due to extensive use of broad spectrum antimicrobials. The lack of uniformity in the use of antibiotics in different geographical areas is likely to result in different resistance rates noted all over the world ${ }^{(12)}$.

Most of the published and running studies for treatment of multidrug-resistant Acinetobacter baumannii depends on the usage of older class of antibiotic (Polymyxins) either alone as a single therapy or in combination with another antibacterial agents. The polymyxins include colistin or polymyxin $\mathrm{E}$ and polymyxin $\mathrm{B}$, and this class of drug has been a savior for the treatment of highly drug-resistant gramnegative bacteria. Colistin is most commonly used in the United States, and it is administered intravenously as a pro-drug known as colistimethate sodium (CMS). A wide range of observational studies have now been published on the clinical efficacy and toxicity of colistin for treating modern day gram-negative bacteria ${ }^{(13)}$. Efficacy

ranges from $\sim 55 \%$ to $80 \%$ depending on the

study and appears to be equal to that of other antibiotics in similar populations. Nephrotoxicity and neurotoxicity remain as key concerns for increasing use in an era of multidrug-resistant pathogens ${ }^{(14)}$.

The problem of associated side effects of colistin especially that critically ill patient have higher incidence of renal impairment which could be aggravated with its usage and the fact that new strains of Acinetobacter baumannii started to show resistance to colistin obliged the investigators to search for other alternatives for treatment. Another major local problem in Egypt is the non availability of colistin in the market which was the triggering point to our team to conduct this research in a trial to test the efficacy of possible alternatives available in the Egyptian market.

The aim of the work was to test the efficacy of combination Doxycycline and cotrimoxazole antibiotics in the treatment of Acinetobacter infection in critically ill patients and its effect on the patient's outcome.

\section{Materials and Methods}

The study was a Prospective Randomized Interventional Trial conducted on 50 adult patients admitted to the Critical Care Medicine Department of Alexandria University Main Hospital in Egyptwith 
multiple drug resistance (MDR) Acinetobacter baumannii (AB) diagnosed from culture and sensitivity test who received combination of doxycycline 100 mg twice daily with Trimethoprimsulphamethoxazole in a dose of $80 \mathrm{mg} / 400$ mg twice daily (modified according to renal functions ) for 7 days.

Patients not tolerating enteral route feeding, Patients with history of drug allergy to Doxycycline or co-Trimoxazole, Patients who do not complete one week therapy with Antibiotics and Patients who received treatment for infections other than A. baumannii were excluded from the study.

Informed consent was taken from all patients or their next of kin. The study was approved from the local ethical committee of the Faculty of Medicine. Follow up culture and sensitivity was repeated after 1 week coverage of antibiotics. Total cure rate as well as cure rate in different subgroups according to the source of infection was followed. Also the mortality related to infection was recorded.

MDR AB was defined as Acinetobacter baumanii that is concomitantly resistant to all the agents of four antibiotic classes (fluoroquinolones, aminoglycosides, cephalosporins, and beta-lactam/betalactamase inhibitor combinations) ${ }^{(15)}$. The microbiological response was considered positive if the organism could not be isolated in repeated samples during or after the course of Antibiotic treatment, and negative if cultures were persistently positive with the same organism 7 days after treatment initiation. Death attributable to primary infection was defined as occurring without resolution of signs and symptoms of infection and with no other cause identified. ${ }^{(16)}$

\section{Results and Discussion}

Table I showed the source of infection in the 50 patients included in the study, 30 of them had respiratory tract infection, 9 patients with surgical site infection, 2 patients with blood stream infection and 9 patients had mixed infections involving more than one site.

Table II showed the total cure rate which was 30 out of 50 patients with a cure rate of $60 \%$ while 20 patients did not show cure. The overall total mortality was 10 out of 50 patients $(20 \%)$ while the infection related death was only 4 patients $(8 \%)$.

Table III showed the cure rate for each category of infection. The highest cure rate was in the surgical site infection 8 out of 9 patients were cured $(88.9 \%)$ while respiratory tract infection subgroup showed a cure of 17 out of 30 patients $(56.7 \%)$. The 2 patients with blood stream infection did not show any cure with a cure rate of $0 \%$. In patients with mixed infections 5 out of 9 patients were cured with a percentage of $(55.6 \%)$

A significant amount of time and energy has been devoted to studying combination therapy for the treatment of A. baumannii infection. Much of the current information is derived from in vitro or animal studies. There are surprisingly limited data from comparative studies involving human A. baumannii infections ${ }^{(17)}$. Petrosillo et al produced a nice review on colistin versus combination therapy; they found only 4 relevant clinical studies and only 1 study demonstrates statistical significance with respect to mortality, favoring monotherapy. The heterogeneity of combinations and infections studied, as well as the small numbers of patients involved, makes interpretation very difficult ${ }^{(14)}$. 
Few published data support the use of doxycycline as a treatment for Acinetobacter baumannii. Goel et al (18) tested the Antibiotic sensitivity pattern of gram negative bacilli isolated from the lower respiratory tract of ventilated patients in the intensive care unit. The sensitivity pattern of Acinetobacter for doxycycline was tested in 38 patients revealing $33.3 \%$ sensitivity, $64.1 \%$ intermediate sensitive organisms while only $2.6 \%$ of Acinetobacter strains were resistant to doxycycline. In another study done by vila $G$ et al ${ }^{(19)}$ testing the In vitro antimicrobial production of betalactamases, aminoglycoside modifying enzymes, chloramphenicol acetyltransferase and susceptibility of clinical isolates of Acinetobacter baumannii. Antimicrobial susceptibility testing was performed on 54 epidemiologically unrelated clinical isolates of Acinetobacter baumannii by using a standard agar dilution technique. On the basis of the in vitro activities, imipenem and doxycycline were the most active agents by $100 \%$ sensitivity to Imipenem and $97 \%$ sensitivity to doxycycline. This was in contrast to a study done by Hernandez et al (20) who studied the effect of doxycycline and other antibiotics alone or in combination for treating lung and blood stream infection of Acinetobacter baumannii in experimental models of mice. No cure rate was seen out of 7 models with lung infection treated with doxycycline alone with only 2 out of 7 $(28.5 \%)$ cured from blood stream infection. With the addition of Amikacin to doxycycline in another 22 models, 3/11 (27\%) of pneumonia models were cured and $11 / 11(100 \%)$ of blood cultures came to be negative for Acinetobacter baumannii. This result denotes clearly the importance of giving the doxycycline as a combination therapy with other antibacterial for treating such serious gram negative infections and that is we had done in our study by the addition of trimethoprim-sulfamethoxazole (co-trimoxazole) to the doxycycline.

Although the usage of co-trimoxazole for treating Acinetobacter baumannii can't be considered as a common or routine practice, yet, some data support a possible curative effect especially when used in combination with other agents. Popescu G.A et al (12) studied the antimicrobial resistance for Acinetobacter baumannii strains, 147 strains of Acinetobacter baumannii were analysed. The main specimen which provided the Acinetobacter baumannii isolates was tracheobronchial aspirate (68 strains, 46.25\%). Less often, other positive samples were: wound secretions (24 strains, $16.32 \%$ ), sputum (18 strains, $12.24 \%$ ), urine (9 strains, $6.12 \%$ ), blood cultures (8 strains, $5.44 \%$ ), removed foreign material ( 7 strains, $4.76 \%$ ), deep collections (4 strains, 2.72\%), cerebrospinal fluid (4strains, $2.72 \%$ ), other pathologic products (5 strains, 3.4\%). The analysis of Acinetobacter baumannii strains' susceptibility for the entire study period revealed a very low susceptibility rate, under $50 \%$ for most of the investigated antibiotics (Tobramycin: $44.45 \%$, Carbapenems: 19.18\%, Amikacin: $18.86 \%$, trimethoprimsulfamethoxazole: $13.77 \%$, fluoroquinolone: 10.89\%, Ampicillin-sulbactam: 9.58\%). Colistin was the only one to prove a high activity $(99.06 \%)$ against these strains. It might be reasonable to claim that the trimethoprim-sulfamethoxazole is not a good choice for treatment but we have to consider that the study tested only the effect of single agent antibiotic which is definitely different from the effect of combinations. Moreover, in the absence of colistin, the results showed that the effect of all other antibiotics is ranging from 10 to $20 \%$ which means that there is no clear superiority to test a combination of any antibiotics over the others. In another study done by $\mathrm{T}$ Karsligil et al ${ }^{(69)}$, the susceptibility of Acinetobacter baumannii to trimethoprimsulfamethoxazole was 47/136 strains which 
represents $34.5 \%$ sensitivity.

The rationale of combination therapy in A. baumannii infections is to reduce the

Table.1 Source of Infection

\begin{tabular}{|l|c|c|}
\hline \multirow{2}{*}{} & \multicolumn{2}{|c|}{ Source of infection } \\
\cline { 2 - 3 } & No & $\mathbf{\%}$ \\
\hline Surgical site & 9 & 18.0 \\
\hline Blood tract & 2 & 4.0 \\
\hline $\begin{array}{l}\text { Respiratory } \\
\text { infection }\end{array}$ & 30 & 60.0 \\
\hline Mixed infection & 9 & 18.0 \\
\hline
\end{tabular}

Table.2 Cure Rate and Mortality

\begin{tabular}{|l|l|l|}
\hline \multirow{2}{*}{} & \multicolumn{2}{|c|}{$\begin{array}{c}\text { Cure rate and } \\
\text { mortality }\end{array}$} \\
\cline { 2 - 3 } & No & $\%$ \\
\hline Cure & & \\
\hline No & 20 & 40.0 \\
\hline Yes & 30 & 60.0 \\
\hline 30 days Mortality & & \\
\hline No & 40 & 80.0 \\
\hline Yes & 10 & 20.0 \\
\hline $\begin{array}{l}\text { Mortality related to } \\
\text { infection }\end{array}$ & 4 & 8.0 \\
\hline
\end{tabular}

Table.3 Relation Between Cure and Site of Infection

\begin{tabular}{|l|l|l|l|l|}
\hline \multirow{2}{*}{ Site of infection } & \multicolumn{4}{|c|}{ Cure } \\
\cline { 2 - 5 } & \multicolumn{3}{|c|}{$\begin{array}{l}\text { No } \\
(\mathrm{n}=\mathbf{2 0})\end{array}$} & \multicolumn{2}{l|}{$\begin{array}{l}\text { Yes } \\
(\mathbf{n}=\mathbf{3 0})\end{array}$} \\
\hline & No & $\%$ & No & $\%$ \\
\hline Surgical site infection & 1 & 11.1 & $\mathbf{8}$ & $\mathbf{8 8 . 9}$ \\
\hline Blood stream & 2 & $\mathbf{1 0 0 . 0}$ & $\mathbf{0}$ & $\mathbf{0 . 0}$ \\
\hline Respiratory tract infection & $\mathbf{1 3}$ & $\mathbf{4 3 . 3}$ & $\mathbf{1 7}$ & $\mathbf{5 6 . 7}$ \\
\hline Mixed infection & 4 & $\mathbf{4 4 . 4}$ & 5 & $\mathbf{5 5 . 6}$ \\
\hline
\end{tabular}

Unfortunately, there are no well designed clinical trials comparing treatment regimens for MDR Acinetobacter infections ${ }^{(9)}$. There is still much debate about the role of combination therapy versus monotherapy for gram negative infections. The results of earlier studies and meta-analyses are difficult to interpret, but more recent evidence is starting to clarify this issue. For empirical treatment, combination therapy improves the likelihood that a drug with in vitro activity against the suspected organism is being administered (often defined as appropriate therapy). This effect is more pronounced in institutions with a greater prevalence of multidrug resistant organisms. 
The antibiotics selected for the combination, however, need to be tailored to local susceptibility data. To our Knowledge, no available data about using the combination studied in the treatment of Acinetobacter baumannii (Doxycycline plus co trimoxazole) ${ }^{(21)}$.

The cure rate in the current study is quiet reasonable in the soft tissue surgical site infection which may be related to better penetration power and I think that this positive result deserves more validation by applying it to larger patients sample especially when there is evidence of colistin resistance. Although the cure rate was zero in blood steam infection but we should interpret this result cautiously as the number of treated patients was only two which make it impossible to reach any strong conclusion and still the use of the combination study can be tried when the colistin is not available. The results related to cure in the respiratory tract infection exceeding the $50 \%$ cure rate cannot be considered bad as the maximum cure rate with the use of colistin as a standard treatment is not far away from this number, I think also that using higher concentration of the two antibiotic used would allow higher penetration power to the lung tissue and definitely will be reflected in the form of higher cure rate but this is can be applied in patients when the risk of serious side effects on them is not very high.

The main limitation related to the relatively smaller number of patients and heterogeneity of infection sites in the current study makes the validation of the results difficult but still it provides a new window for exploring more probable combinations for better cure of Acinetobacter baumannii infections in critically ill patients.

\section{References}

1. Juni E,1978. Genetics and physiology of Acinetobacter. Annu Rev Microbial. 32:349-351.

2. Lee JC, Koerten, H, van den Broek P,2006. Adherence of Acinetobacter baumannii strains to human bronchial epithelial cells. Res Microbial; 157:360363.

3. Joly-Guillou, ML,2005. Clinical impact and pathogenicity of Acinetobacter. Clin Microbial Infect; 11:868-870.

4. Wang, SH, Sheng, WH, Chang, YY, 2007.Healthcare-associated outbreak due to pan-drug resistant Acinetobacter baumannii in a surgical intensive care unit. J HospInfect ; 53:97-99.

5. El Shafie SS, Alishaq M, Leni Garcia, M,2004. Investigation of an outbreak of multidrug-resistant Acinetobacter baumannii in trauma intensive care unit. $\mathbf{J}$ Hosp Infect; 56:101-103.

6. Siegman-Igra Y, Bar-Yosef, S, Gorea, a, Avram, J,2010. Nosocomial Acinetobacter meningitis secondary to invasive procedures: report of 25 cases and review. ClinInfect Dis; 17:843-847.

7. Gordon NC, Wareham DW,2010. Multidrug-resistant Acinetobacter baumannii : mechanisms of virulence and resistance. Int $\mathbf{J}$ AntimicrobAgents ; 35:219.

8. Bishburg E, Bishburg K, 2009. Minocycline-an old drug for a new century: emphasis on methicillin-resistant Staphylococcus aureus (MRSA) and Acinetobacter baumannii. Int $\mathrm{J}$ Antimicrob Agents; 34(5):395-401.

9. Jang $\mathrm{T}$, Lee $\mathrm{S}$, Huang $\mathrm{C}$, Lee $\mathrm{C}$, Chen $\mathrm{W}, 2009$. Risk factors and impact of nosocomial Acinetobacter baumannii blood stream infections in the adult intensive care unit: a case-control study. J Hosp Infect; 73: 143-50.

10. Mahgoub S, Ahmed J, Glatt AE, 2002. Underlying characteristics of patients harboring highly resistant Acinetobacter 
baumannii. Am J Infect Control; 30(7):386-90.

11. Popescu G. A., Gavriliu Liana-Cătălina, Streinu-Cercel A, 2011. Antimicrobial resistance of Acinetobacter baumannii strains isolated in „MATEI BALŞ” national institute of infectious diseases. Therapeutics, Pharmacology and Clinical Toxicology. 15(3): 225-9

12. Peleg AY, Seifert H, Paterson DL, 2008. Acinetobacter baumannii : emergence of a successful pathogen. ClinMicrobiol Rev; 21(3):538-82.

13. Falagas ME, Kasiakou SK,2005. Colistin: the revival of polymyxins for the management of multidrug-resistant gramnegative bacterial infections. Clin Infect Dis; 40(9):1333-41.

14. Petrosillo N, Ioannidou E, Falagas ME, 2008. Colistinmonotherapy vs. combination therapy: evidence from microbiological, animal and clinical studies. Clin Microbiol Infect. 14(9):81627.

15. Maragakis LL, Perl TM, 2008. Acinetobacter baumannii : epidemiology, antimicrobial resistance, and treatment options. Clin Infect Dis; 46(8):1254-63.

16. Falagas ME, Koletsi PK, Bliziotis IA, 2006. The diversity of definitions of multidrug-resistant (MDR) and pandrugresistant (PDR) Acinetobacter baumannii and Pseudomonas aeruginosa. J Med Microbiol. 55:1619-29.

17. NidhiGoel, Uma Chaudhary, Ritu Aggarwal, KiranBala, 2009. Antibiotic sensitivity pattern of gram negative bacilli isolated from the lower respiratory tract of ventilated patients in the intensive care unit. Indian Journal of Critical Care Medicine; 13(3): 148-51.

18. Vila J, Marcos A, Marco F, Abdalla S, Vergara Y, Reig R, Gomez-Lus R, Jimenez de Anta T, 1993.In vitro antimicrobial production of betalactamases, aminoglycoside-modifying enzymes, and chloramphenicol acetyltransferase by and susceptibility of clinical isolates of Acinetobacter baumannii. Antimicrob Agents Chemother. 37(1):138-41.

19. Maria Hernandez, Jeronimopachon, Cristina Pichardo etal, 2000. Imepenem, Doxycycline and Amikacin in monotherapy and in combination in Acinetobacter baumannii experimental pneumonia. Journal of Antimicrobial Chemotherapy;45: 493-501.

20. T Karsligil, I Balci and Y Zer, 2004. Antibacterial Sensitivity of Acinetobacter Strains Isolated from Nosocomial Infections. The Journal of International Medical Research; 32: 436 - 44.

21. Holloway KP, Rouphael NG, Wells JB, King MD, Blumberg HM, 2010. Polymyxin B and doxycycline use in patients with multidrug-resistant Acinetobacter baumannii infections in the intensive care unit. Expert Opin Pharmacother. 11(5):779-88.

\section{How to cite this article:}

Farid, S., A. Abouelela and Eliwa, M. 2016. Doxycycline and Co-trimethoxazole: A new combination for treatment of MDR Acinetobacter baumannii. Does it work? Int.J.Curr.Microbiol.App.Sci. 5(1):157-164. doi:'http:///dx.doi.org/10.20546/ijemas.2016.5011.013 